\title{
Ein elektrostatischer Brückenindikator ${ }^{1}$
}

\author{
Von THEOdor GAST \\ Aus dem Institut für technische Physik der Technischen Hochschule Darmstadt 2 \\ (Z. Naturforschg. 2 a, 344-348 [1947]; eingegangen am 28. Februar 1947)
}

Die Verbindung eines flächenhaft entarteten Saitenelektrometers mit einer im Ausschlagverfahren arbeitenden Hochfrequenzbrücke stellt ein neues Meßprinzip dar. Dieses ermöglicht die Umformung von elektrischen Größen, wie Strom, Spannung und Widerstand, oder die Verwandlung der Ergebnisse von Rechenoperationen mit diesen Größen, wie Produkt und mittelbar auch Quotient bzw. Leistung und Phasenwinkel, in proportionale Ströme. Sie können angezeigt und registriert werden oder zur Steuerung selbsttätiger Meß- und Regeleinrichtungen dienen. Damit ist es möglich, Wechselstrombrücken in einem weiten Frequenzbereich nach zwei Komponenten selbsttätig abzugleichen.

\section{Elektrostatische Umformung von Meßwerten}

$\mathrm{B}$ ei Arbeiten mit einer sehr empfindlichen Meßbrücke für Tonfrequenz ${ }^{3}$ mit dem Ziel, dielektrische Sättigungserscheinungen aufzuzeigen, ergab sich folgende Störung: Die hohen Gleichspan-

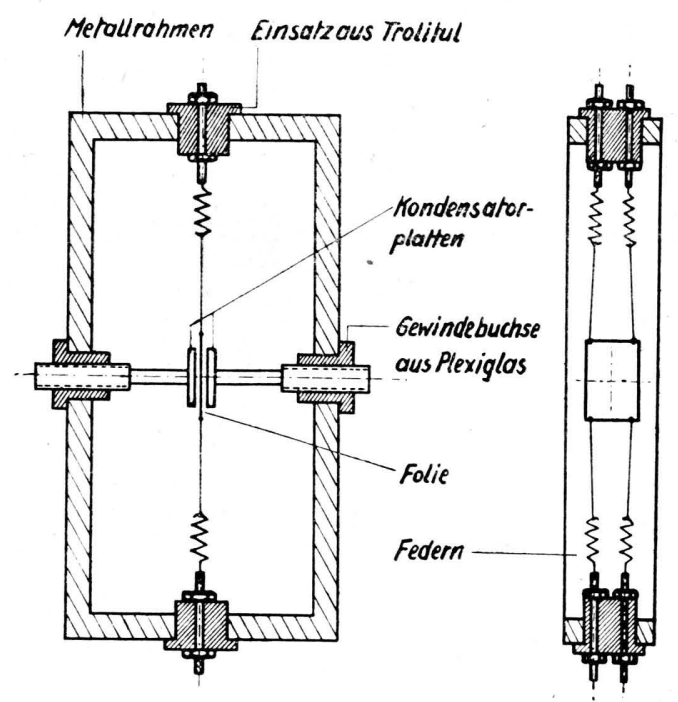

Abb. 1. Das Folienelektrometer im Schnitt.

nungen, die hierbei in den Meßkreis eingeführt werden mußten, änderten die Kapazität der Vergleichskondensatoren meßbar und gesetzmäßig, und zwar infolge elastischer Verformung durch

1 Teilauszug aus der Habilitationsschrift „Selbstabgleich und Registrierung in der physikalischen Feinmeßtechnik unter besonderer Berücksichtigung der technischen Physik der Kunststoffe" und Weiterführung der ihr zugrunde liegenden Gedanken. elektrostatische Kräfte. Es war demnach möglich, die angelegte Gleichspannung durch Kapazitätsmessung zuverlässig zu ermitteln, und man durfte annehmen, daß eine abgewandelte und auf diesen Meßzweck ausgerichtete Anordnung zur genauen und praktisch leistungsfreien Messung auch sehr kleiner Gleichspannungen geeignet sein müßte.

Diese Vermutung wurde wie folgt bestätigt:

Die in Abb. 1 gezeigte Anordnung, bestehend aus einem Plattenkondensator, zwischen dessen Elektro-

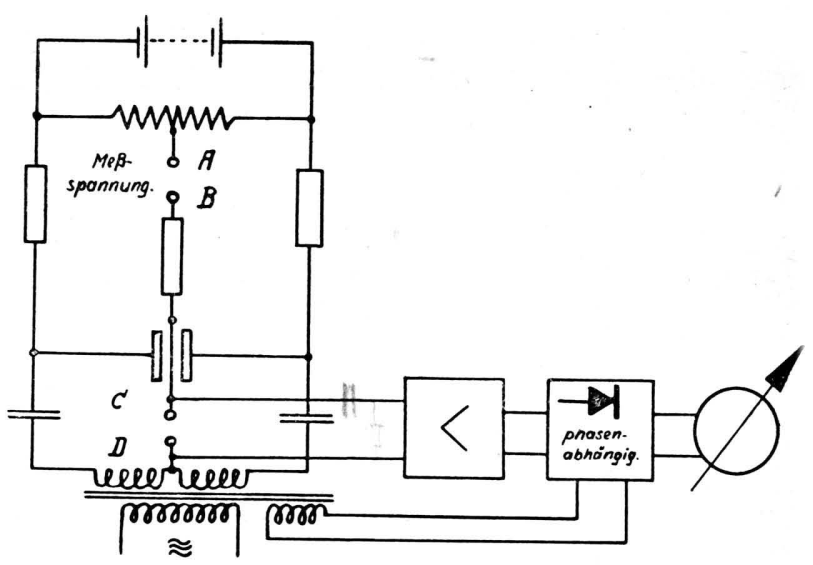

Abb. 2. Verwendung des Elektrometers zur Messung kleiner Gleichspannungen.

den eine leitende Folie federnd und isoliert aufgehängt ist, bildet den oberen Zweig der in Abb. 2 dargestellten Hochfrequenzbrücke. Die Brückendiagonale ist an den Eingang eines Hochfrequenzverstär-

${ }^{2}$ Hrn. Prof. Dr. R. Vi ew eg schulde ich Dank für die Bereitstellung der Forschungsmittel, für die Förderung der Arbeit unter schwierigen Bedingungen und für wertvollen Rat.

${ }^{3}$ R. Vieweg u. Th. Gast, Z. techn. Physik 24. 56 [1943]. 
kers angelegt. Die Ausgangsspannung wird phasenabhängig gleichgerichtet und gemessen. Diese Spannung ist in Grenzen ein lineares Maß für die Auslenkung der Folie. Sie hat in der Symmetrielage den Wert Null und ändert sinngemäß ihr Vorzeichen, wenn sich die Folie von einer Seite durch die Mitte zur anderen hinüber bewegt. Der Folienkondensator ist außerdem mit einer Gleichspannungsquelle und einem Spannungsteiler zu einer Elektrometerschaltung vereinigt, wie sie vom Wulfschen Einfadenelektrometer bekannt ist. Die Folie ändert ihre Lage je nach Größe und Richtung der zwischen $A$ und $B$ angelegten Meßspannung. Dadurch wird die Hochfrequenzbrücke verstimmt, und das Instrument im Ausgang spricht an. Gleichstromteil und Wechselstrombrücke sind durch Kondensatoren bzw. Widerstände voneinander abgeriegelt.

Orientierende Versuche mit dieser Anordnung ergaben bei befriedigender Nullpunktskonstanz eine Empfindlichkeit von $6 \mathrm{~mA} / \mathrm{V}$. Es gelang mit Hilfe eines Tintenschreibers von $3 \mathrm{~mA}$ Endausschlag und $3000 \mathrm{Ohm}$ Meßwerkwiderstand, Isolationsströme der Größenordnung 10-13 A zu registrieren und so z. B. den Einfluß von Feuchtigkeit und Oberflächenbehandlung bei Kunststoffen bequem und schnell zu verfolgen. Ferner erwies es sich als möglich, einen konstanten Widerstand in den Stromkreis des Anzeigegeräts zu schalten und mit dem resultierenden Spannungsabfall die Eingangsspannung teilweise zu kompensieren, so daß die Konstanz des Übersetzungsverhältnisses Ausgangsstrom: Eingangsspannung verbessert und die Nullpunktssicherheit erhöht wurde. Weiter lag es nahe, die Anwendung des beschriebenen Elektrometers auch auf das Wechselstromgebiet auszudehnen und seine Eigenart als produktbildendes Meßelement sowohl im Hinblick auf phasenempfindliche Anzeige als auch zur Leistungsmessung auszuwerten. Für den Vorgang, der jedem Wert der zu messsenden Größe, sei es Strom, Spannung, Widerstand, Leistung oder Phasenwinkel, eine bestimmte Stromstärke im Ausgang der Meßeinrichtung zuordnet, scheint der Ausdruck „statische Meßwertwandlung“ geeignet zu sein. Im folgenden wird über die rechnerischen Grundlagen dieser Meßwertwandlung berichtet.

2. Rechnerische Grundlagen der Mefwertumwandlung mit dem Folien-

\section{elektrometer}

Wir bezeichnen die an den Kondensatorplatten liegende Spannung mit $U$, die Teilspannungen zwischen Folie und Platten mit $U_{l}$ und $U_{r}$, die zu messende Spannung mit $V$, den Abstand der Platten mit $l$, die Teilabstände nennen wir $l_{l}$ und $l_{r}$, die Auslenkung der Folie $a$, ihre Rückstellkraft $c$; die Fläche der einzelnen Platte heiße $F$ und die Dielektrizitätskonstante $\varepsilon$ (Abb. 3). Die Folie erfährt von links und rechts Anziehungskräfte.

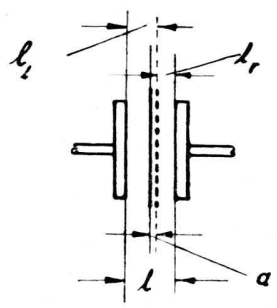

Abb. 3. Bezeichnung der Abstände im Folienkondensator.

$$
\begin{aligned}
& P_{l}=\frac{U_{l}^{2}}{l_{l}^{2}} \frac{\varepsilon F}{8 \pi}=\left(\frac{U}{2}+V\right)^{2} \frac{1}{l_{l}^{2}} \frac{\varepsilon F}{8 \pi}, \\
& P_{r}=\frac{U_{r}^{2}}{l_{r}^{2}} \frac{\varepsilon F}{8 \pi}=\left(\frac{U}{2}-V\right)^{2} \frac{1}{l_{r}^{2}} \frac{\varepsilon F}{8 \pi} .
\end{aligned}
$$

Nun bewirkt die Differenz dieser Kräfte

$$
P=P_{l}-P_{r}
$$

eine Auslenkung

$$
a=P / c=\frac{\varepsilon F}{8 \pi c}\left\{\frac{\left(\frac{U}{2}+V\right)^{2}}{\left(\frac{l}{2}-a\right)^{2}}-\frac{\left(\frac{U}{2}-V\right)^{2}}{\left(\frac{l}{2}+a\right)^{2}}\right\} .
$$

Unter der Voraussetzung: $V \ll U$ und $a \ll l$ läßt sich dies vereinfachen $\mathrm{zu}$

$$
a \approx \frac{\varepsilon F}{\pi c} \frac{U^{2}}{l^{2}}\left\{\frac{V}{U}+\frac{a}{l}\right\},
$$

und es folgt

$$
\frac{a}{l} \approx \frac{V}{U}\left\{\frac{1}{\pi \frac{c l^{3}}{\varepsilon F U^{2}}-1}\right\} .
$$

Hierin sind $U$ und $V$ in elektrostatischen Einheiten, $l$ und $a$ in $\mathrm{cm}, c$ in dyn/cm und $F$ in $\mathrm{cm}^{2}$ einzusetzen. $\varepsilon$ wird im allgemeinen gleich 1 sein, sofern man nicht Dämpfung durch eine isolierende Flüssigkeit vorsieht. Die Voraussetzung $a \ll l$ bringt Beschränkungen in der Wahl der Größenverhältnisse von $c, U$ und $l$. Praktisch ist für zu kleines $c$ die Einstellung nicht mehr stabil. Durch grundsätzliche Änderung der Elektrodenanordnung dürfte es indessen möglich sein, Stabilität auch ohne mechanische Rückstellkräfte zu erreichen. Damit wäre die in (1) angedeutete Möglichkeit zur Quotientenmessung gegeben.

Bei relativ großer Rückstellkraft gilt $\pi \frac{c l^{3}}{\varepsilon F U^{2}} \gg 1$, und Gl. (1) vereinfacht sich zu

$$
\frac{a}{l} \approx-\frac{V U \varepsilon F}{\pi c l^{3}} .
$$


Hieraus geht hervor, daß für gegebenes $l$ die Auslenkung $a$ eine Funktion des Produktes $V \cdot U$ ist. In diesem Fall wird das Elektrometer zum Produktmeßgerät.

Nun besteht die Rückstellkraft aus einem mechanischen Teil, der durch die Aufhängung der Folie gegeben ist, und einem elektrostatischen, der von dem Einfluß der hochfrequenten Hilfsspannung herrührt und folgendermaßen zustandekommt:

Wird die Folie aus ihrer Nullstellung ausgelenkt, dann tritt zwischen $C$ und $D$ (Abb.2) bei abgetrenntem Verstärker eine Hochfrequenzspannung der Größe

$$
\Delta U_{H F}=U_{H F} \frac{a}{l}
$$

auf, worin $U_{H \boldsymbol{F}}$ die an den Kondensatorplatten liegende hochfrequente Hilfsspannung ist. Belastet man mit dem Eingangswiderstand $Z_{E}$ des Verstärkers, so verringert sich die Spannung zwischen $C$ und $D$ um den Betrag

$$
\Delta U_{C D}=\Delta U_{H F}\left(1-\frac{Z_{E}}{Z_{E}+Z_{J}}\right) .
$$

Hierin bedeutet $Z_{J}$ den zwischen $C$ und $D$ gemessenen Wechselstromwiderstand der Brücke. $\Delta U_{C D}$ ist gleich der Differenz zwischen dem Potential der Folie und dem ihrem Ort entsprechenden Potential im Kondensator und bewirkt eine rücktreibende Kraft der Größe .

$$
P=-\frac{\varepsilon F}{8 \pi}\left(\frac{U_{H F}^{3}}{l}\right)\left(1-\frac{Z_{E}}{Z_{J}}\right) \alpha .
$$

Der Faktor von $a$ bedeutet eine ,elektrostatische Federkonstante", die in der Direktionskraft $c$ enthalten ist.

3. Das Folienelektrometer als Brückenindikator

Gl. (2) sagt aus, daß $a / l$ dem Produkt der Spannungen $V$ und $U$ proportional ist. Handelt es sich hierbei um sinusförmige Wechselspannungen, dann gilt:

$$
\frac{a}{l}=V_{0} \sin \omega t U_{0} \sin (\omega t+\varphi),
$$

worin $\varphi$ der Phasenwinkel ist. Die Dämpfung der Folie und die Trägheit der Abstandsmeßanordnung bewirken, daß am Anzeigegerät der zeitliche Mit- telwert über die Periode

$$
\frac{1}{T} \int_{0}^{T} \frac{\alpha}{l}=\frac{1}{T} \int_{0}^{T} V_{0} U_{0} \sin \omega t \sin (\omega t+\varphi) d t
$$

erscheint. Hierfür ergibt sich

$$
\frac{1}{T} \int_{0}^{T} \frac{a}{l}=\frac{V_{0} U_{0}}{2} \cos \varphi=V_{\mathrm{eff}} U_{\mathrm{eff}} \cos \varphi .
$$

Das Elektrometer ist also phasenempfindlich und somit als Leistungsmesser für Wechselstrom geeignet. Für die Wirkungsweise ist es dabei gleichgültig, ob die kleinere Spannung $V$ zwischen der Folie und dem Symmetriepunkt der größeren Spannung $U$ an den Platten angelegt wird, oder ob um-

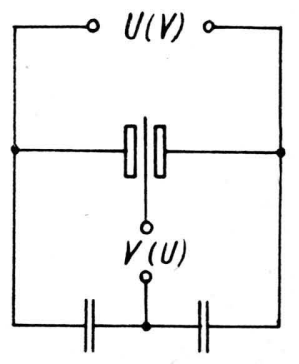

Abb. 4. Vertauschbarkeit von Hilfs- und Meßspannung.

gekehrt die größere Spannung $U$ zwischen die Folie und den Symmetriepunkt der an die Platten angelegten kleinen Spannung zu liegen kommt. In beiden Fällen ist die Feldstärke in den beiden Kondensatorhälften durch den Einfluß von $V$ verschieden groß, und dies veranlaßt eine Verschiebung der Folie (Abb.4). Hier wie dort führt die quadratische Abhängigkeit der Kraft von der Feldstärke zu gemischt linearen Gliedern mit $U$ und $V$ und damit zur Produktbildung. Die in Abb. 4 angegebene Symmetrieschaltung leitet zwanglos zur Wechselstrombrücke über, als deren Indikator das Folienelektrometer infolge seiner Frequenzunabhängigkeit, seiner Phasenempfindlichkeit, der Vertauschbarkeit von $U$ und $V$ und der Eignung zu Fernanzeige, Registrierung und Steuerung eine Fülle neuer Möglichkeiten bringt.

Als Beispiel sei die in Abb. 5 gezeigte Schaltung herausgegriffen. Wir haben eine Vierkapazitätenmeßbrücke vor uns, in deren Diagonale das Folienelektrometer eingeschaltet ist. Die Folie liegt zunächst an dem oberen Brückeneckpunkt. In die Zuleitungen zu Folie und Kondensatorplatten sind 
Schwingungskreise eingeschaltet, die auf die Frequenz der Hilfsspannung abgestimmt und mit einem Hochfrequenzgenerator und der Anzeigevorrichtung induktiv gekoppelt sind. Befindet sich die Brücke im Gleichgewicht, dann ist die Folie kräftefrei. Vergrößert man nun z. B. den Wert von $C_{1}$, dann wird die linke Teilspannung des Elektrometers kleiner, und die Folie bewegt sich nach rechts. Hierdurch tritt im Ausgang des Hochfrequenzverstärkers eine Spannung auf, deren Größe der Kapazitätsänderung in Grenzen proportional ist. Durch Abgleich von $C_{1}$ kann der Ausschlag des Anzeigegerätes rückgängig gemacht und die

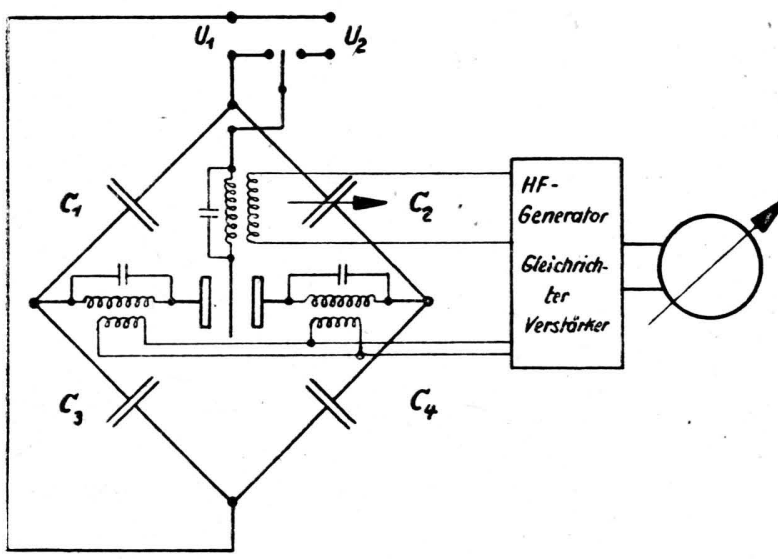

Abb. 5. Das Folienelektrometer als Brückenindikator. $U_{1}=$ Brückenspannung, $U_{2}=$ phasenverschobene Hilfsspannung.

Verstimmung beseitigt werden. Ändern sich hingegen bei gleichbleibender Kapazität die dielektrischen Verluste von $C_{1}$, so ist die entstehende Diagonalspannung in ihrer Phase gegenüber der Spannung zwischen Folie und Brückenmitte um $90^{\circ}$ verschoben, und das Instrument spricht nicht an. Legt man aber die Folie nicht an den oberen Brückeneckpunkt, sondern an eine Hilfsspannung $U_{2}$, deren Vektor, von dem Potential der Brückenmitte an gerechnet, auf dem Vektor der Brückenspannung senkrecht steht, dann reagiert das Elektrometer nur auf dielektrische Verluste und nicht mehr auf kapazitive Verstimmungen.

Die Anordnung eignet sich daher wie die unter ${ }^{3}$ beschriebene Meßbrücke mit B r a u n schem Rohr als Indikator zu getrenntem Abgleich von Kapazität und dielektrischem Verlust. Während dort die Meßfrequenz auf $800 \mathrm{~Hz}$ festgelegt war, ist sie hier

4 F. W e idmann, Kunststoffe 29, 133 [1939].

5 R. Vieweg u. Th. Gast, Kunststoffe 34, 221 [1944]. nach unten grundsätzlich nicht und nach oben durch die Höhe der zur Abstandsmessung benutzten Hilfsfrequenz $\left(\nu=3 \cdot 10^{6}\right)$ begrenzt. Legt man die Hilfsfrequenz verhältnismäßig tief $\left(\nu=10^{5}\right)$, so besteht bei geeigneter Auslegung der Kopplungsglieder die Möglichkeit, Brückenmessungen mit getrenntem Abgleich nach Kapazität und Verlust im Hochfrequenzgebiet durchzuführen, wobei die Hilfsfrequenz die untere Grenze festlegt, während die obere nurmehr durch die geometrischen Abmessungen des Systems bestimmt wird.

4. Selbsttätiger Abgleich von Meßbrükken mit Hilfe des Folienelektrometers

Es ist bekannt, daß die Verwendung des Einfadenelektrometers als Brückeninstrument bei der Messung hoher Widerstände Vorteile bietet ${ }^{4}$. Das Folienelektrometer läßt sich infolge seines analogen Aufbaus in der gleichen Weise wie das Einfadenelektrometer in die Widerstandsmeßbrücke einschalten. Es hat dabei den Vorzug objektiver Ablesung an einem Zeigerinstrument. Darüber hinaus läßt sich der Ausgangsstrom des Verstärkers zur Betätigung eines Hilfsmotors ausnützen, der den Brückenabgleich selbsttätig vornimmt. Wenn man mit dem vom Motor gesteuerten Brükkenteil ein Schreibwerk verbindet, so dürfte es möglich sein, Isolationswiderstände zuverlässig zu registrieren.

Selbsttätige Wechselstrombrücken sind in verschiedenen Ausführungsformen entwickelt wor$\operatorname{den}^{3,5,6,7}$. Sie arbeiten mit festen Betriebsfrequenzen, deren Änderung selbst in engen Grenzen schwierig ist. Das in der vorliegenden Arbeit behandelte Meßprinzip macht es indessen möglich, Selbstabgleich nach zwei Komponenten und Registrierung von Kapazität und dielektrischem Verlustfaktor in einem sehr breiten Frequenzbereich durchzuführen und damit Kurven der Dielektrizitätskonstante und der dielektrischen Verluste von Isolierstoffen in Abhängigkeit von Frequenz, Temperatur und anderen Variablen unmittelbar aufzuzeichnen.

Dies konnte für die kapazitive Komponente bereits im Versuch bestätigt werden (Abb.6). Die Anordnung wurde hierbei so getroffen, daß an dem Ausgang des Verstärkers ein Gleichstrom-Nullmotor geschaltet ist, der über ein. Getriebe den

${ }^{6}$ W. Ge y g e r, Arch. Elektrotechn. 31, 115 [1934].

7 Amerikanisches Schrifttum, Nachweis durch Brand in Verlust geraten. 


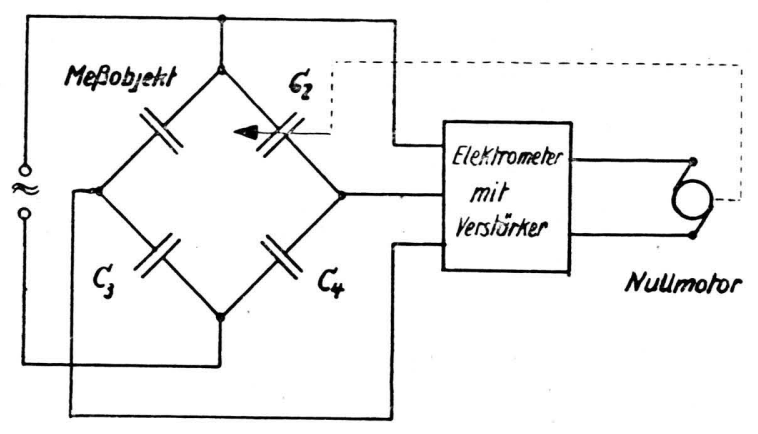

Abb.6. Selbsttätiger Kapazitätsabgleich.

Drehkondensator $C_{2}$ bewegt. Der Meßbereich erstreckt sich von $10 \mathrm{pF}$ bis $200 \mathrm{pF}$. Der Abgleich vollzieht sich im Bruchteil einer Sekunde. Das Drehmoment reicht aus, um nötigenfalls außer dem
Drehkondensator noch ein Schreibwerk zu betätigen und dabei eine Ansprechempfindlichkeit von $0,1 \mathrm{pF}$ zu gewährleisten. Untersuchungen an weichgestelltem Polyvinylchlorid, einem in der Niederspannungstechnik vielfach verwendeten Isolierstoff, ergaben, und zwar zunächst qualitativ, die erwartete Abnahme der Dielektrizitätskonstanten mit steigender Frequenz. Durch Parallelschalten Ohmscher Widerstände $\mathrm{zu}$ dem verlustarmen Kondensator $C_{2}$ konnte ferner gezeigt werden, daß diese Maßnahme den Kapazitätsabgleich so lange nicht stört, als für den resultierenden Verlustfaktor $\operatorname{tg} \delta \ll 1$ gilt. Versuche über den automatischen $\mathrm{Ab}$ gleich dielektrischer Verluste sind in Vorbereitung.

Frl. Dipl.-Phys. Erika Alpers danke ich für wertvolle Hilfe bei Berechnung und Bau der Hochfrequenzgeräte.

\title{
Mischungsentropie und osmotischer Druck von Lösungen langgestreckter, starrer Teilchen (Zur statistischen Theorie makromolekularer Lösungen II $^{1}$ )
}

\author{
Von G. V. Schulz \\ Aus dem Institut für physikalische Chemie der Universität Mainz \\ (Z. Naturforschg. 2 a, 348-357 [1947]; eingegangen am 22. Dezember 1946)
}

\begin{abstract}
Statistische Rechnungen zeigen, daß die Mischungsentropie und damit der osmotische Druck in Lösungen langgestreckter Teilchen beträchtlich höher als in solchen kugelförmiger Teilchen ist. Dieses kommt dadurch zustande, daß sich langgestreckte Teilchen in ihrer räumlichen Orientierung gegenseitig behindern, und zwar um so stärker, je höher die Konzentration ist. Der Effekt ist allein vom Achsenverhältnis der Teilchen abhängig. Die entsprechenden Gleichungen werden für ein zylinderisches Modell abgeleitet.

Das Achsenverhältnis der Serumglobulin- und der Myosinmoleküle ist aus Messungen der Sedimentationsgeschwindigkeit in der Ultrazentrifuge bekannt (Kabat u. Peder sen; H. H. Weber). Auch aus den osmotischen Messungen von Burk u. Greenbẹg bzw. H. H. W eber läßt sich nach den neu abgeleiteten Gleichungen das Achsenverhältnis berechnen. Die verschiedenen Methoden führen zu befriedigend übereinstimmenden Ergebnissen.
\end{abstract}

$\mathrm{I}_{\mathrm{s}}^{\mathrm{n}}$ den letzten Jahren ist eine Reihe von Versuchen gemacht worden, die in Lösungen makromolekularer Stoffe mit Fadenmolekülen auftretenden starken Abweichungen vom van t'H offschen Gesetz vom Standpunkt der statistischen Mechanik aus zu erklären. Den Rechnungen wurde das Gittermodell der Lösung zugrundegelegt; danach enthält die Lösung eine Anzahl

${ }^{1}$ I. Mitteilung dieser Reihe: G. V. Schulz, Z. Naturforschg. 2a, 27 [1947]. - Die Bezeichnungen der vorliegenden Arbeit sind die gleichen wie in der früheren Arbeit. festliegender Gitterpunkte, deren jeder entweder von einem Lösungsmittel-Molekül oder einem Grundmolekül des gelösten Stoffes besetzt wird. Das Problem wird in der Weise gelöst, daß man die Anzahl der Anordnungsmöglichkeiten (thermodynamische Wahrscheinlichkeit) ausrechnet, die für $N_{1}$ Moleküle des Lösungsmittels und $N_{2}$ Moleküle des gelösten Stoffes (zu je $n$ Grundmolekülen) bestehen.

Den ersten theoretisch einwandfreien Ansatz in dieser Richtung machten Fowler und Rush- 